\title{
Digital Image Watermarking using DWT-SVD HF Technique
}

\author{
Jyoti Kumari \\ Dept. of Electronics \& \\ Communication \\ SISTec, Bhopal, India
}

\author{
Pankaj Vyas \\ Dept. of of Electronics \& \\ Communication \\ SISTec, Bhopal, India
}

\begin{abstract}
In this research work adopted the frequency domain watermarking scheme which is embedded using discrete wavelet transform (DWT) singular value decomposition (SVD) and High Boost Filtering (HF). By singular values factoring it represent smaller set of values and it can preserve constructive feature of an original image. After that, apply high boost filtering in decomposed in high frequency sub-band on both images to improve the value PSNR. The MSE, PSNR and NC performance parameters are taken to measure the efficiency of the propose method. The simulated experimentation is done in MATLAB and the simulation results of propose method (DWT-SVD-HF) gives improved results than the existing method (DWT).
\end{abstract}

\section{Keywords}

DWT, SVD, High Boost Filter, Digital Image Watermarking. MATLAB, PSNR

\section{INTRODUCTION}

Because of the current advancement in web technology, redistribution of computerized substance has turned out to be simple. It prompts to the intense need of sheltered and real condition for the handling of advanced substance. This downside can be overcome by utilizing watermarking technology. Watermarking of images has as of late obtained enormous interest in a scope of applications like, identification of image, copyright protection, verification of image and information stowing away, among others. Duplication and dissemination of sight and sound information have been rendered simple and for all intents and purposes costless because of huge advances in systems administration and high speed processors. Digitized information can without much of a stretch be controlled along these lines losing its inventiveness [1].Thus it makes copyright security of advanced media a stern challenge. In this way the idea of advanced watermarking comes into picture. Advanced watermarking is the handle that implants information called a watermark into a sight and sound protest in a manner that the watermark can be later on recognized or extricated for a question declaration purposes. The sight and sound articles, in which the watermark is inserted, are as a rule called the first, cover flag, have flag or basically the work [2].The watermark ought to be inserted in such a way, to the point that the inventiveness of the host picture ought not be twisted [3].A advanced watermark is a recognizing snippet of data that is appointed to the information to be secured. One imperative necessity by this is the watermark can't be effectively extricated or expelled from the watermarked project.

A powerful digital watermarking technique must fulfill the two primary prerequisites of impalpability and robustness to basic images attacks like trimming, revolution, Gaussian clamor, movement obscure, salt and pepper clamor, pressure and numerous more flag preparing operations. Advanced picture watermarking strategies are assembled into spatial and frequency domain.

A. Spatial Domain

- It is manipulating or changing an image representing an object in space to enhance the image for a given application.

- Techniques are based on direct manipulation of pixels in an image

- Used for filtering fundamentals, smoothing filters, sharpening filters, unsharp masking and laplacian

B. Frequency Domain

- This technique are based on modifying the spectral transform of an image

- It transform the image to its frequency representation

- Carry out an image processing

- Figure out inverse transform back to the spatial domain

- High frequencies correspond to pixel values that modify hastily across the image (e.g. text, texture, leaves, etc.)

- Strong low frequency components correspond to huge scale features in the image (e.g. a single, homogenous object that dominates the image)

The image watermarking processing is shown in fig.1. In this research, we proposed DWT-SVD - HF technique to watermark the images which improve the quality and security of image.

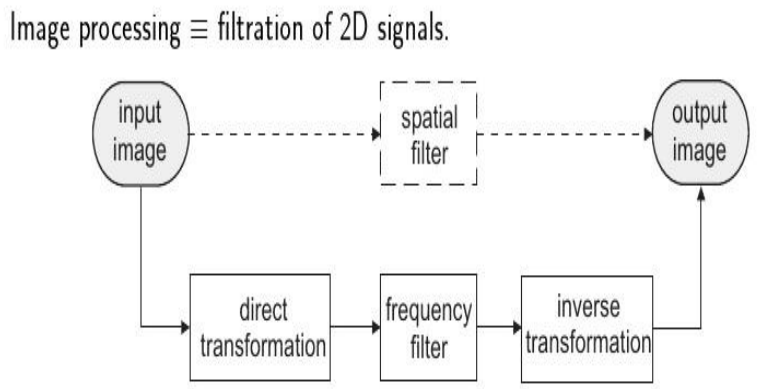

Fig.1: Digital Image Watermarking

The simulation and analysis of the propose method is done using MATLAB simulation toolbox and performance evaluation is done among PSNR, MSE and NC parameters. The experimental results of our propose method give improved result than existing method. The remaining part of the research paper is done as follows: 
Section 2 presents the literature about the image watermarking - Section III gives brief explanation about watermarking technique. Proposed methodology is described in Section IV. Section V presents the experimental results and analysis and last section present brief conclusion of the research work.

\section{RELATED WORK}

In [4] cover image is decomposed into low and high frequency components by the application of 1-level DWT (Discrete Wavelet Transform). Average of each sub-band is premeditated. The watermark is embedded into the 1-level high-high, high-low, and low high sub-band of cover image using AP (Arithmetic Progression) technique. The sub-band which has the negligible average is to be embedded initial. Afterward, the watermarked image is projected to numerous attacks like median filtering, JPEG compression, Gaussian lowpass filtering, shearing, cropping, rotation etc. with different distortion strengths. The watermark which is embedded in the middle frequency sub-bands and high frequency sub-band is taken out by analogous mechanism. The imperceptibility and robustness of the watermarked image is checked out by measuring the PSNR (Peak Signal to Noise Ratio) and SSI (Structural Similarity Index) values. From the implementation results, they came to know that this watermarking algorithm can withstand numerous image manipulations compared to other existing DWT based methods. In [5] developed an imagewatermarking method is to which persuade both imperceptibility and robustness requirements. To accomplish this objective, a fusion image-watermarking scheme based on discrete wavelet transform (DWT) and singular value decomposition (SVD) is anticipated in this paper. In their method, the watermark is not embedded straight on the wavelet coefficients but rather than on the elements of singular values of the cover image's DWT sub-bands. Experimental consequences are provided to demonstrate that the proposed method is able to withstand a variety of image-processing attacks.

In [6] projected a technique to embed fractal images in discrete wavelet transform (DWT). The binary watermark image is engendered from fractal codes. The color cover image is separated into its grayscale equivalents. The grayscale counterparts of color image are used to embed the watermark image which is engendered from fractal codes in standard frequency blocks to guard the codes from attacker. Counterparts watermark images are embedded into grayscale equivalents disjointedly to enhance the robustness and security of the system. The consequence analysis showed that the robustness and imperceptibility of the algorithm.

In [7] introduced a novel scheme to safeguard digital images' copyrights. That's why, as ISB method was selected in relation to the mechanism in an endeavor overpower the concerns of robustness and imperceptibility in watermarked metaphors. According to the literature analysis, embedding the aimed surreptitious bits (Watermark) is a challenging apprehension within a host image (ordinary 8-bit, grey-scale) in a sense to assemble it imperceptible by the HVS (Human Visual System) other than the substance that it is predicted to accept any attacks. The recommended method here correspond to an improved method for the embedding of ISB which safeguards the robustness and cultivates the rate of sanctuary by employing repeated bits in different bit planes over an irregular order and it extends the LSB system exclusively in circumstances where robustness and imperceptibility are major concerns of assessment.
In [8] adopted the usage of a mixed (hybrid) transformation to accomplish these objectives, The outlook behind applying a fusion transform or mixed transformation is that the cover image is personalized in its singular values rather than on the DWT sub-bands and also PSNR values of cooperation cover image and watermark can be transform, consequently the watermark makes it susceptible to vivid attacks and preserves its original state by checking the robustness. To maintain the methods and comparative study some simulation results were presented.

\section{WATERMARKING TECHNIQUE}

In digital image processing, different watermarking techniques has been developed in which some of them we are describing below:

\section{A. Discrete Wavelet Transform (DWT)}

Discrete wavelet transform is applied to decompose any nonstationary signal like an image, audio or video signal. The transform is predicated on little waves, known as wavelets, of varying frequency and limited duration. Frequency as well as spatial information of an image is retained during wavelet transformation. Temporal information is preserved during this conversion method [9]. Wavelets are made by translations and dilations of constant function called mother wavelet. DWT is performed by low-pass and high-pass filtering of an image. High-pass filter creates detailed image pixels and low-pass filter creates coarse approximation image pixels [10]. The outputs are down-sampled by 2 after performing the low-pass and high-pass filtering. 2D DWT is done by executing 1D DWT on each row, which is known as horizontal filtering and then on each column, which is known as vertical filtering [11].

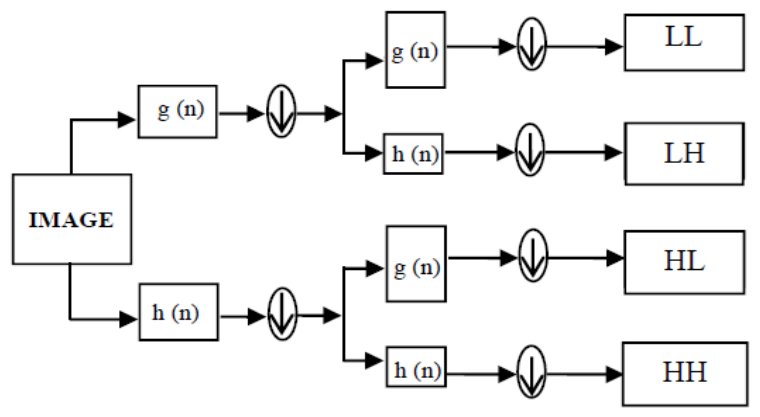

Fig.2: 2D-DWT decomposition of an input image using filtering approach

\section{B. Singular Value Decomposition}

SVD (Singular value decomposition) produces the purview of minimization of intricacy by segmenting the digital image matrix which is not negative into $\mathrm{U} * \mathrm{~S} * \mathrm{VT}$, At this point orthogonal matrices are $\mathrm{U}$ and $\mathrm{V}$ and orginary matrix has singular values which are orderly in reducing order [12]. $\mathrm{S}$ is the diagonal matrix of the image, for instance, when any disarrange is done on the digital image enormous diversity in the singular values does not betide. Singular values also demonstrate actual algebraic features [13]. Singular value decomposition is a numerary scheme which is used in numerical assess for in diagonal matrix. For numerous of applications singular value decomposition is evolve as an algorithm. In image processing applications singular value decomposition has various features. SVDs or singular values decomposition of digital image have astonishing indelible, for instance, when any disarrange is done on the digital image huge diversity in the singular values do not betide. The singular value decomposition (SVD) is used to un-ridden diverse 
numerical issues in linear algebra. The watermarking which is based on SVD method, numerous inlets are feasible. In original image's high frequency band SVD is imposed, which is mostly used inlet and embed the watermark information to modify the singular values. The noteworthy feature of singular value decomposition is when gigantic of the tampered singular values transform that is incredibly small for numerous types of attacks [14].

\section{High Boost Filtering}

High boost filtering [15] can be implemented with one pass using either of the two masks shown in equation (1), (2). Note that, when $\mathrm{A}=0$, high-boost filtering becomes "standard" Laplacian sharpening. As the value of A amplifies past 1, the involvement of the sharpening process becomes less and less important. Eventually, if A is large enough, the high-boost image will be approximately equal to the original image multiplied by a constant.

$$
\begin{aligned}
& {\left[\begin{array}{ccc}
0 & -1 & 0 \\
-1 & A+4 & -1 \\
0 & -1 & 0
\end{array}\right]} \\
& {\left[\begin{array}{ccc}
-1 & -1 & -1 \\
-1 & A+8 & -1 \\
-1 & -1 & -1
\end{array}\right]}
\end{aligned}
$$

One of the principal applications of High Boost filtering is when the input image is darker than desired.

By varying the boost coefficient, it generally is possible to obtain an overall increase in average gray level of the image, thus helping to brighten the result.

In this paper, a customary type of High Boost filter is used. It is revealed in the $3 \times 3$ convolution matrix looks as follows:

$$
\left[\begin{array}{ccc}
-1 & -1 & -1 \\
-1 & 9 & -1 \\
-1 & -1 & -1
\end{array}\right]
$$

\section{PROPOSED METHODOLOGY}

In this section of the research work, we describes our propose methodology for image watermarking which uses DWT-SVD$\mathrm{HF}$ technique.

\section{Singular Value Decomposition (SVD)}

Singular value decomposition (SVD) is a theory of linear algebra. In this approach it can transform into $R=\left(S_{a} V_{a} D_{a}\right)^{T}$ where it can refractor any digital image into three separate matrices. By singular values factoring it represent smaller set of values and it can preserve constructive feature of an original image. SVD method factors R into three matrices S, V, D i.e.

$R=\left(S^{*} V^{*} D\right)^{T}$.

Here $S$ is an $m 1 \times n l$ orthogonal matrix

$\boldsymbol{D}$ is an $m 2 \times n 2$ orthogonal matrix

$\boldsymbol{V}$ is complete $m \times n$ diagonal matrix.

The complete diagonal matrix is singular values.

The advantage of SVD over in digital watermarking is value has interstices algebraic property in first whereas matrix size is not rigid in secondly and at finally singular values in images are not affected on small perturbation under attacks.

A novel approach based on DWT-SVD with hybrid filter under various attacks.

\section{Embedding process steps:}

Suppose

$\boldsymbol{A}(\boldsymbol{i}, \boldsymbol{j})$ is cover image of size and square images. Shown in fig 1 (a)

$\boldsymbol{B}(\boldsymbol{i}, \boldsymbol{j})$ is a watermark secret image. Shown in fig 1(b)

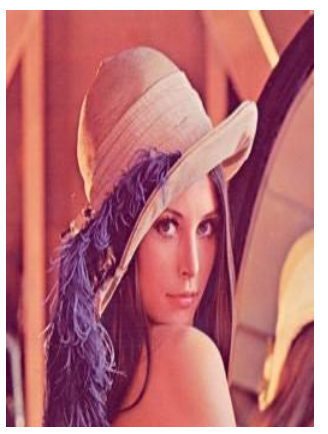

\section{City of Lakes}

\section{Bhopal}

Fig. 3: (a) Cover Image

(b) Secret watermark image

2. Apply two level wavelet transformations into both cover $\boldsymbol{A}(\boldsymbol{i}$, $\boldsymbol{j})$ and secret watermark $\boldsymbol{B}(\boldsymbol{i}, \boldsymbol{j})$ images, it will divide into subbands i.e. LLLL, LLLH, LLHL, LLHH.

3. Apply hybrid boost filter in decomposed high frequency subband on both images and obtained result into $\boldsymbol{h} \boldsymbol{f}(\boldsymbol{i}, \boldsymbol{j})$.

4. Perform IDWT $^{-1}$ on LLHH sub band on both decomposed images.

5. Now decompose the both cover and secret watermark images HH sub-bands using singular transform.

$$
\begin{aligned}
& H_{h f}=S_{h f} V_{h f} D_{h f} \\
& H_{h}=S_{b} V_{b} D_{b}
\end{aligned}
$$

6 . Then modify the singular value of $h f$ image with the singular value of secret watermark image:

$\mathbf{V}_{\mathbf{b}}=\left(\mathbf{V}_{\mathbf{h f}}+\boldsymbol{\alpha}\left(\mathbf{V}_{\mathbf{b}}\right)\right)$, here $\boldsymbol{\alpha}$ is a watermarked strength value.

7. Find the modified $\mathbf{H H}$ sub band of $h f$ image as

$$
\mathbf{H H}_{\mathrm{hf}}=\mathbf{S}_{\mathrm{hf}} \mathbf{V}_{\mathrm{hf}} \mathbf{D}_{\mathrm{hf}}
$$

8. Now again apply DWT on $\mathbf{H H}_{\mathrm{hf}}$ sub-band obtain $\mathbf{H H}_{\mathbf{h}}$ * as resultant image.

9. Apply IDWT ${ }^{-1}$ using all decomposed sub-bands LLPerform inverse discrete wavelet transform using $\mathbf{L} \mathbf{L}_{\mathbf{h f}}, \mathbf{L H}_{\mathbf{h f}}, \mathbf{H L}_{\mathbf{h f}}$ and $\mathbf{H H}_{\mathrm{hf*}}$ to achieve targeted watermarked image $\boldsymbol{w m}(\boldsymbol{i}, \boldsymbol{j})$.

10. Store watermarked image separately into disk.

\section{Watermarking extraction process}

The main prospective of this approach is to obtain secret watermark image which will be similar to original secret image. This technique is semi-blind scheme where original image is not required for obtaining the secret watermark and cover image.

1. Apply two level DWT on the watermarked image $\boldsymbol{w m}(\boldsymbol{i}, \boldsymbol{j})$ as $\mathbf{L L}_{\mathrm{WMi}}, \mathbf{L H}_{\mathrm{WMi}}, \mathbf{H L}_{\mathrm{WMi}}, \mathbf{H H}_{\mathrm{WMi}}$

2. Apply IDWT ${ }^{-1}$ on high frequency $\mathbf{H H}_{\mathbf{W M i}}$ sub-band of secret watermark image.

3. Apply SVT into the $\mathbf{H H}$ band of watermarked image $\boldsymbol{w m}(\boldsymbol{i}$, $\boldsymbol{j})$, secret watermark image and hybrid $\boldsymbol{h} \boldsymbol{f}(\boldsymbol{i}, \boldsymbol{j})$ image as in embedding process, then go to step 4 . 
4. Start extraction process, singular value of watermark image from HH sub-band as:

$\mathbf{V}_{\mathrm{WMi}}=\left(\left(\mathbf{V}_{\mathrm{WMi}}-\mathbf{V}_{\mathrm{hf}}\right) / \boldsymbol{\alpha}\right)$

5. Get the improved high frequency $\mathrm{HH}$ band as:

HH $_{\text {WMi* }^{*}}=\mathbf{S}_{\mathrm{b}} \mathbf{V}_{\text {WMi }} \mathbf{D}_{\mathrm{b}}$

6. Apply DWT on improved high frequency $\mathrm{HH}_{\mathrm{WM}^{*}}$ band of watermark image as: $\mathbf{H H}_{\mathrm{WMi}^{* *}}=\mathbf{D W T}\left(\mathbf{H H}_{\mathbf{W M i}}{ }^{*}\right)$

7. Apply IDWT ${ }^{-1}$ using $\mathbf{H H}_{\mathbf{W M}} \mathbf{i}^{* *}$ and also in remaining three sub-bands to extract the secret watermark image as $\boldsymbol{o w m}(\boldsymbol{i}, \boldsymbol{j})$.

8. Apply hybrid boost filter into $\boldsymbol{o w m}(\boldsymbol{i}, \boldsymbol{j})$ to remove noise from it and enhanced obtained secret image.

9. Now apply $\mathbf{M S E}=\frac{\mathbf{1}}{\mathbf{M N}} \sum \sum\left(\mathbf{W}_{\mathbf{i j}}-\mathbf{H}_{\mathbf{i j}}\right)^{2}$ formula to obtain MSE.

10. Then Apply $\boldsymbol{P S N R}=\mathbf{1 0}_{\log }\left(\frac{\mathbf{2 5 5 ^ { 2 }}}{\mathbf{M S E}}\right)$ formula to obtain PSNR.

\section{EXPERIMENTAL OUTCOMES}

The experimental analysis of the proposed methodology is done using a widely used MATLAB2012A toolbox and the machine configuration is Intel $\mathrm{I}_{3}$ core $2.20 \mathrm{Ghz}$ processor, with 4GB RAM, windows 7 home basis. In proposed methodology we applied a DWT-SVD-HF technique to improve the quality and security over digital image and for comparative analysis of the propose method is perform on PSNR, MSE and NC.

\subsection{Snapshot}

The simulation of the proposed methodology (DWT-SVD with HF) and existing method (DWT) is applied on the image dataset of Baboon, Barbara, Cameraman and Lena image. The snapshot of these images after simulation is illustrated below. After simulation the image quality of proposed method is better than the existing method.
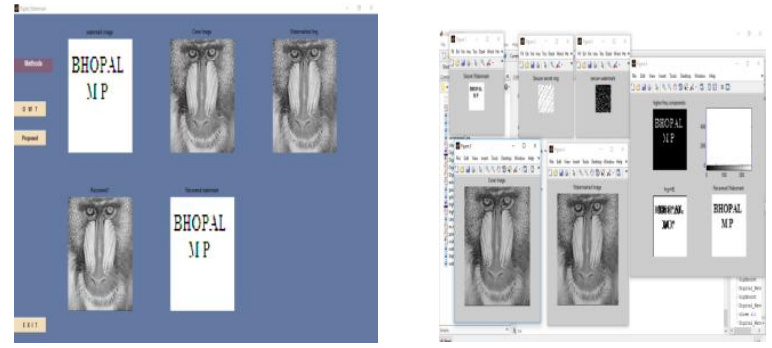

(a) Snap of baboon image using DWT (b) Snap of baboon image using DWT-SVD-HF

Fig.4: Snapshot of Baboon Image dataset
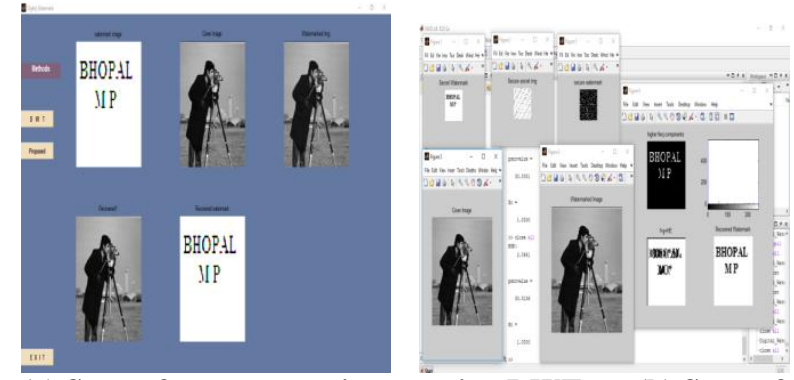

(a) Snap of cameraman image using DWT

(b) Snap of cameraman image using DWT-SVD with HF

Fig.5: Snapshot of Cameraman Image dataset
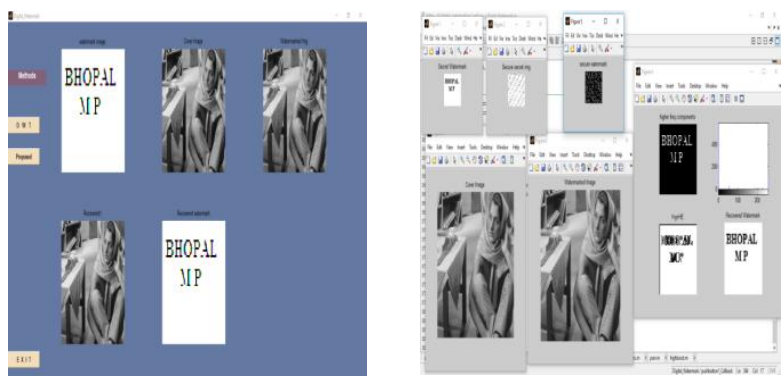

(a) Snap of barbara image using DWT

(b) Snap of barbara image using DWT-SVD with HF

Fig.6: Snapshot of Barbara Image dataset
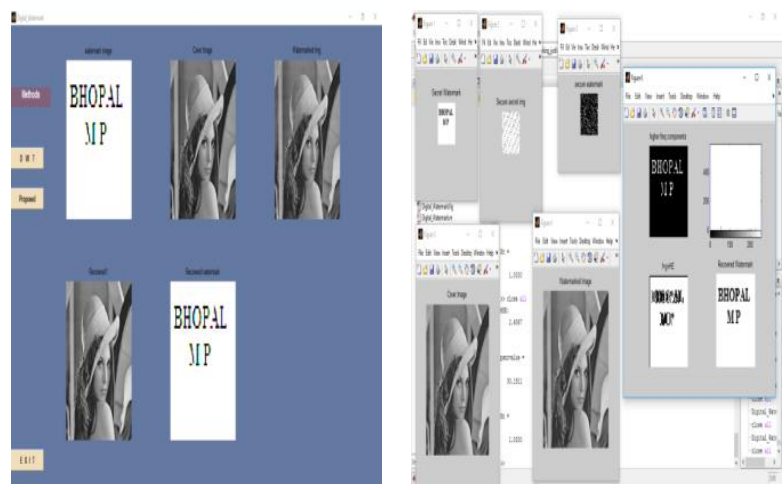

(a) Snap of Lena image using DWT (b) Snap of Lena image using DWT-SVD with HF

Fig.7 Snapshot of Lena Image dataset

\subsection{Result Analysis}

There are various performances measuring parameter for the images datasets are available but in this dissertation we mainly use the PSNR (Peak Signal to Noise Ratio), MSE (Mean Square Error) and NC (Normalized Coordinates). The comparative analysis of proposed method and existing method for MSE parameters is done and it is found that the our methodology give better results than the existing method about $7-8 \%$.

Table 1: MSE Comparison

\begin{tabular}{|l|c|c|}
\hline Image & DWT & DWT-SVD-HF \\
\hline Lena & 5.9956 & 2.7300 \\
\hline Barbara & 5.6879 & 2.8103 \\
\hline Baboon & 7.998 & 2.4716 \\
\hline Cameramen & 3.5982 & 2.0641 \\
\hline
\end{tabular}




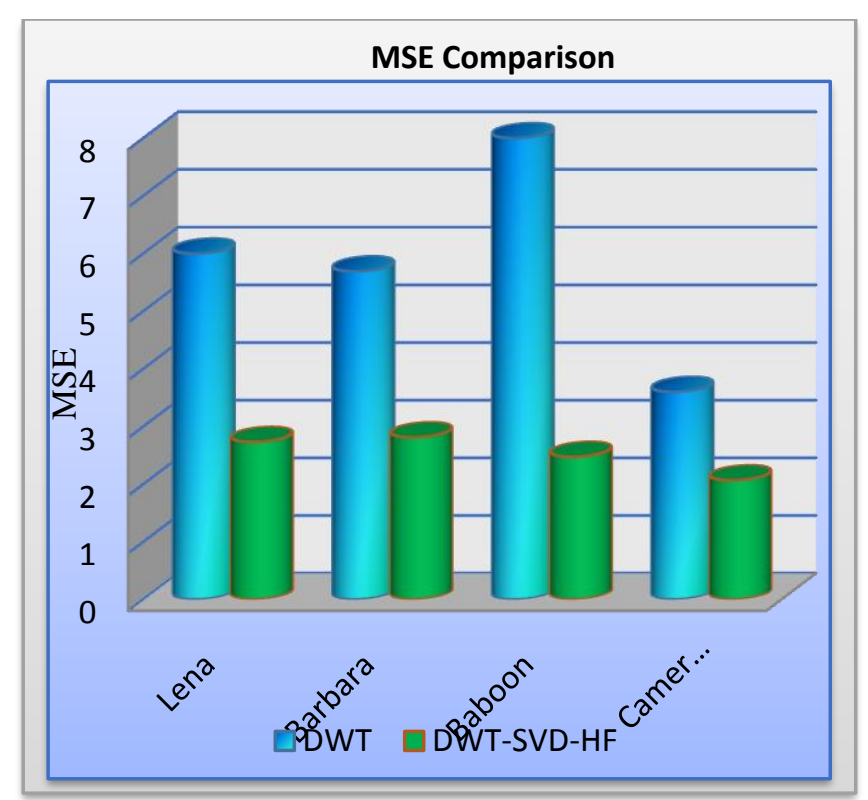

Fig.8: Comparative analysis of DWT-SVD-HF and DWT method for MSE

Similarly the comparative analysis of the proposed method and existing method is performed on PSNR parameter. In image processing, is usually used to assess the differences in the degree of image quality, from preprocessing to post-processing. A larger value of PSNR means there is a little difference between original image and processed image. A PSNR value greater than or equal to 30 means that the processed image quality is acceptable and the value of proposed is more than the value of existing method.

Table 2: PSNR Comparison

\begin{tabular}{|l|c|c|}
\hline Image & DWT & DWT-SVD-HF \\
\hline Lena & 29.81197 & 30.1911 \\
\hline Barbara & 24.45459 & 30.0051 \\
\hline Baboon & 22.32058 & 29.9737 \\
\hline Cameramen & 26.75537 & 30.5136 \\
\hline
\end{tabular}

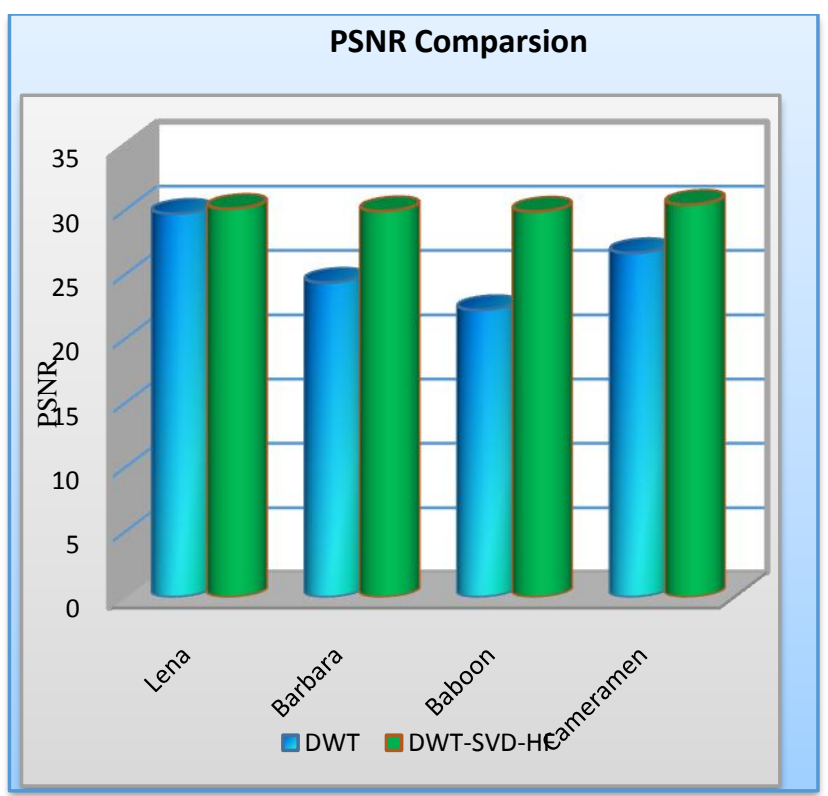

Fig.9 Comparative analysis of DWT-SVD-HF and DWT method for PSNR

The performance analysis is performed between exiting and proposed method for the normalized coordinated (NC) parameter in which we found that the simulation result of our method is improved than the existing method which is near about $100 \%$. This method utilize the orientation information provided by normalization while using as little of the normalized domain as possible.

Table 3 NC Comparison

\begin{tabular}{|l|c|c|}
\hline Image & DWT & DWT-SVD-HF \\
\hline Lena & 0.931211 & 1 \\
\hline Barbara & 0.864733 & 1 \\
\hline Baboon & 0.687885 & 1 \\
\hline Cameramen & 0.953285 & 1 \\
\hline
\end{tabular}

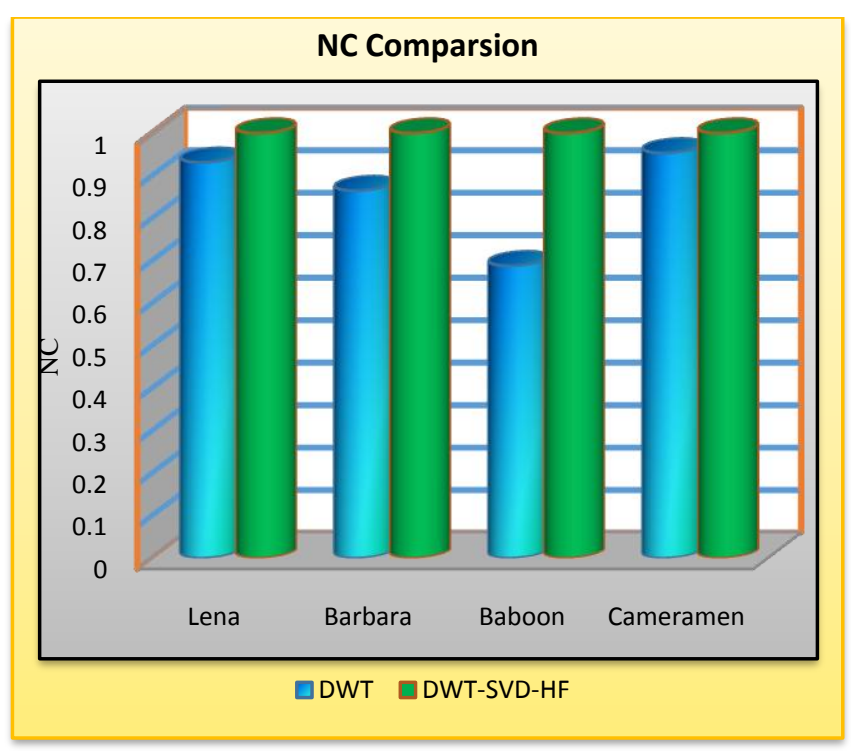

Fig.10 Comparative analysis of DWT-SVD-HF and DWT method for $\mathrm{NC}$ 


\section{CONCLUSION}

In this dissertation, we studied the different watermarking algorithm such as spatial domain and frequency domain. In contrast to the spatial-domain-based watermarking, frequencydomain-based techniques can embed more bits of watermark and are more robust to attack. Online application of watermarking for video in the spatial domain becomes cumbersome due to associated high computational complexities involved. On the other hand, Watermarking in the DCT domain needs preprocessing operations such as inverse entropy coding and inverse quantization. In this thesis we embed DWT, SVD and High boost filter technique to watermark the digital image efficiently. Watermarking algorithms have varied requirements according to the application, the algorithm aims to target. Three such requirements have been dealt with in the dissertation they are PSNR (Peak Signal to Noise Ratio), MSE (Mean Square Error) and NC (Normalized Coordinate). The experimental analysis is performed on these parameters and it is analyzed that our propose method (DWT-SVD-HF) outperforms than the exiting method (DWT). It means that this propose method provide efficiently watermark the image and enhances the quality of it.

In future work, will attempt to abolish the need to save information and therefore make the detection mechanism completely blind. Another improvement of the method would be to accomplish more vigorous feature points allowing for more watermarks to be detected.

\section{REFERENCES}

[1] R.G.van Schyndel , A.Z. Tirkel , and C.F.Osborne, A digital watermark, in proc. IEEE int. conf. image processing (ICIP), 1994.

[2] X. Xia C.Boncelet and G.Arce , A multiresolution watermark for Digital images ,Proc IEEE int. conf. on image processing (ICIP), Oct 1997.

[3] Barni M. Bartolini F, Piva , An improved wavelet based watermarking through Pixel wise Masking , IEEE transactions on image processing, 2001.

[4] Meeta Malonia, Surendra Kumar Agarwal "Digital Image Watermarking using Discrete Wavelet Transform and Arithmetic Progression Technique", 2016 IEEE Students' Conference on Electrical, Electronics and Computer Science.

[5] Chih-Chin Lai, and Cheng-Chih Tsai "Digital Image Watermarking Using Discrete Wavelet Transform and Singular Value Decomposition", IEEE Transactions on Instrumentation And Measurement, Vol. 59, No. 11, November 2010
[6] Arun , Kabi, K.K. ; Saha, B.J. ; Pradhan, C. "Enhanced digital watermarking scheme using fractal images in wavelets", Computing, Communication and Networking Technologies (ICCCNT), 2014 International Conference on 11-13 July 2014, Page(s):1 - 6 Print ISBN:978-14799-2695-4.

[7] Sharifara A., Rahim, M.S.M. and Bashardoost, M. "A Novel Approach to Enhance Robustness in Digital Image Watermarking Using Multiple Bit-Planes of Intermediate Significant Bits", Informatics and Creative Multimedia (ICICM), 2013 International Conference on 4-6 Sept. 2013. Published in IEEExplore, Page(s):22 - 27.

[8] Jaishri Guru Hemant Dhamecha Brajesh Patel, "Fusion of DWT and SVD digital watermarking Techniques for robustness", International Journal of Advanced Research in Computer Science and Software Engineering. Volume 4, Issue 9, September 2014 ISSN: 2277 128X.

[9] R. Kaur, and S. Jindal, "Robust digital watermarking in high frequency band using median filter function based on DWT-SVD," International Conference Advanced Computing and Communication Technology, pp. 47-52, Feb 2014.

[10] I. J. Cox, M. L. Miller, J. A. Bloom, J. Fridrich and T. Kalker, "Digital watermarking and steganography," San Mateo, CA, USA:Morgan Kaufmann, 2008.

[11] E. Nezhadarya, Z. J. Wang, R. K. Ward, "Robust image watermarking based on multiscale gradient direction quantization," IEEE Trans. Image Process., vol. 6, no. 4, pp. 1200-1213, 2011.

[12] Q. Li, C. Yuan, and Y.-Z. Zhong, “Adaptive DWT-SVD domain image watermarking using human visual model," in Proc. 9thInt. Conf. Adv. Commun. Technol., GangwonDo, South Korea, pp. 1947-1951, 2007.

[13] S. Mallat, "The theory for multiresolution signal decomposition: The wavelet representation," IEEE Trans. Pattern Anal. Mach. Intell., vol. 11, no. 7, pp. 654-693, Jul. 1989.

[14] Sangeeta Madhesiya, Shakil Ahmed, "Advanced Technique of Digital Watermarking based on SVD-DWTDCT and Arnold Transform", International Journal of Advanced Research in Computer Engineering \& Technology (IJARCET) Volume 2, No 5, May 2013.

[15] Gonzalez RC, Woods RE. Digital Image Processing: Introduction; 2002. 